\title{
Le Domérien-Toarcien Inférieur De La Ride De Fert Elbir (Rides Sud-Rifaines, Maroc)
}

\author{
Mohamed Erragragui \\ Aïssa Masrour \\ Hicham Benbaqqal \\ Mostafa Gretaa
}

Equipe " Géosciences, Patrimoine et Substances utiles»

Université Moulay Ismail, Faculté des Sciences, Département de géologie, Meknès

Doi: 10.19044/esj.2017.v13n36p132 URL:http://dx.doi.org/10.19044/esj.2017.v13n36p132

\begin{abstract}
The South-Rifain ridges are located at $30 \mathrm{Km}$ in the North of Meknes City, they constitute the extreme southern limit of the Rifaine chain. Globally, these ridges integrate into the history of African northwestern margin evolution. Mainly, the frame of this ridge is composed of the Jurassic sedimentation. The Fert Elbir ridge, subject of this study, is among the leading reliefs in the South-Rifain ridges. Generally, in this ridge, two geological sections have been studied, in the objective to determine and to understand the history of sedimentological, bio-stratigraphical and paleoenvironmental evolution of the Jurassic sedimentary series. Essentially, it includes the carbonates and marls deposits, composed mainly by several facies and micro-facies, grouped in eight facies associations. They contain a very important biological diversity especially at the level of the middleDomerian. Paleo-environmentally, this sedimentation series was deposited in the many diversified environments, having the areas of deposit essentially very close to the coast (proximal), or located in distal position with several hundred meters from the shore.
\end{abstract}

Keywords: South-rifain Ridges, Carbonate, Palaeoenvironment, Jurassic, Domerian-Toarcian

\section{Résumé}

Les rides sud-rifaines sont situées à $30 \mathrm{Km}$ au Nord de la ville de Meknès. Elles constituent la limite méridionale de la chaine rifaine. Elles s'intègrent dans l'histoire de l'évolution de la marge africaine nordoccidentale pendant le Jurassique. L'ossature de ces rides est composée par 
des formations sédimentaires jurassiques. La ride de Fert Elbir, objet de cette étude, est l'un des principaux reliefs des rides sud-rifaines. Deux coupes géologiques de cette ride ont été effectuées, pour appréhender l'évolution sédimentologique, bio-stratigraphique et paléoenvironnementale de la série sédimentaire du Domérien-Toarcien inférieur. Le cortège sédimentaire est composé essentiellement par des dépôts carbonatés et marneux qui sont regroupés en huit associations faciologiques. Elles renferment une diversité biologique très importante surtout au niveau du Domérien moyen. De point de vue paléoenvironnement, ces faciès sont déposés dans plusieurs types d'environnements, comportant essentiellement des milieux de dépôts proximaux très proches du littoral ou des milieux distaux.

Mots-clés : Rides sud-rifaines, Carbonate, Paléoenvironnement, Jurassique, Domérien-Toarcien

\section{Introduction}

Le Jurassique marocain a inauguré une période de grandes transgressions marines caractérisées principalement par une sédimentation faiblement détritique, déposée dans des bassins subsidents, à fond instable à cause d'anciennes failles hercyniennes, qui ont joué en décrochement ou en extension. Il s'agit généralement des bassins du Haut-Atlas, du Moyen-Atlas, des rides sud-rifaines et de la région de Melillia (Saaidi, 1983). Dans les rides sud-rifaines, la succession sédimentaire de l'intervalle Trias-Paléocène s'est déposée et accumulée principalement dans des bassins délimités par un système de failles normales de direction NE-SW (Sani et al, 2007).

Dans l'objectif de la compréhension de l'évolution sédimentologique, bio-stratigraphique et paléoenvironnementale de la série sédimentaire du Domérien-Toarcien inférieur de ces rides, l'investigation sera menée sur deux coupes géologiques de la ride de Fert Elbir : la coupe du Jbel Bin Bou Skif et la coupe du Jbel Abd Elkrim.

\section{Cadre géologique et géographique}

Les rides sud-rifaines affleurent sous forme de deux arcs parallèles et concaves vers le Nord-Est (arc oriental et arc occidental), séparés par le plateau d'El Gaada. Elles sont limitées par l'Oued Beht à l'Ouest, l'Oued Sebou à l'Est, le complexe Pré-rifain au Nord et la plaine de Saïs au Sud. La ride de Fert Elbir (fig.1), définie par Daguin (1927) et la S.C.P (1952), se située dans la partie nord-ouest du massif de Zerhoun, à $30 \mathrm{Km}$ vers le Nord de la ville de Meknès, juste à côté de la ville de Moulay Idriss Zerhoun et le site antique de Volubilis.

les Rides sud-rifaines affleurent entre deux domaines structuraux très différents : le domaine Rifain au Nord, qui fait partie de la chaîne Alpine de 
la Méditerranée occidentale, édifiée principalement au Miocène supérieur et le domaine méséto-atlasique au Sud, structuré à l'ère primaire et secondaire. Ces rides sont encadrées par des bassins à remplissage néogène et quaternaire, qui résultent de la déformation Mio-pliocènes (Vernli, 1987 ; Boutakiout, 1990 ; Ait Brahim, 1991). De point de vue structural, elles sont le résultat de la migration des déformations tectoniques depuis le domaine interne du Rif vers son domaine externe. La compression régionale ENEWSW de la phase Miocène supérieure a provoqué le raccourcissement du socle paléozoïque et le décollement de sa couverture au niveau du Trias (Haddaoui, 2000).

Sur le plan paléo-environnemental, cette région est prédominée par une plate-forme carbonatée durant le Jurassique inférieur et moyen (Faugeres, 1978).

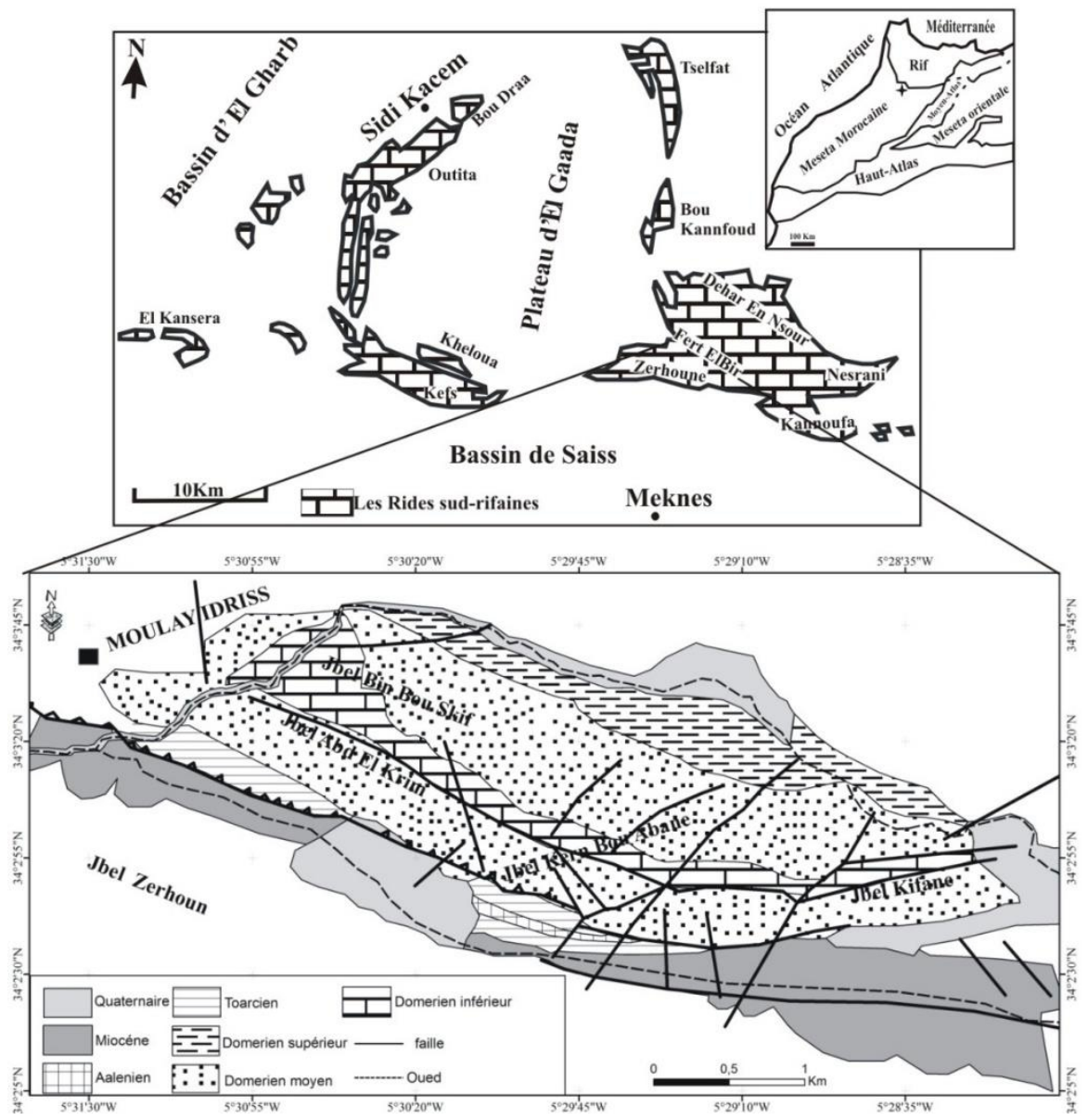

Figure 1 : Schéma géologique et situation géographique de la ride de Fert El Bir (d'après la carte géologique de Beni Ammar 1/50000 ; Chenakeb 1991-1995). 


\section{Matériel et Méthode}

Sur le terrain, chaque coupe géologique a fait l'objet d'une étude de la succession sédimentaire du Domérien-Toarcien inférieur d'un point de vue lithologique, sédimentologique, paléontologique et tectonique. L'ensemble des bancs qui affleurent ont fait l'objet d'un échantillonnage détaillé.

$\mathrm{Au}$ laboratoire, le matériel prélevé sur le terrain a fait l'objet de plusieurs analyses paléontologiques et pétrographiques. Pour l'examen microscopique, une trentaine des lames minces ont été confectionnées sur des roches essentiellement carbonatées. L'étude des lames est basée sur la terminologie de Purser (1980) et Lucas et al. (1976); alors que la détermination de la nature des éléments figurés est basée sur la classification de Folk (1959) ; Dunham (1962) ; Embry et Klovan (1971) a servi comme référence pour l'étude de l'organisation texturale de ces éléments et son interprétation hydrodynamique. L'interprétation paléoenvironnementale est basée sur plusieurs travaux (Masrour, 2003 ; Flügel, 2004 et 2010 ; ElfAquitaine, 1975 et 1977 et Wilson, 1975).

\section{Description litho-stratigraphique}

Les coupes géologiques de la ride de Fert Elbir sont réalisées selon un transect SE-NW, depuis Jbel Abd Elkrim jusqu'au Jbel Bin Bou Skif. La formation du Domérien supérieur a été relevée principalement plus vers le NE, dans la partie basale du Jbel Kern Bou Abane et Jbel El Kifane. Elles sont composées essentiellement par la série sédimentaire de l'intervalle Domérien-Toarcien inférieur. La série est constituée de formations carbonatées et marneuses, regroupées en huit associations de faciès. Ces faciès renferment une diversité biologique très importante surtout au niveau du Domérien moyen.

\subsection{Coupe géologique du Jbel Abd Elkrim}

Jbel Abd Elkrim est situé au Sud de la ride de Fert Elbir. La coupe géologique (fig.2) a été relevée dans le versant Sud. Globalement, ce versant anticlinal en pente raide, affleure sur plusieurs dizaines de mètres, du haut vers le bas on distingue trois formations sédimentaires ; celle du Domérien inférieur, moyen puis celle du Toarcien inférieur.

\section{a- Formation du Domérien inférieur}

Cette formation constitue l'importante falaise perchée qui prédomine le sommet du Jbel Abd Elkrim. Elle affleure sous forme d'une centaine de mètres de calcaire lité, caractérisé par une stratification sub-verticale et une déformation tectonique très intense, avec des failles normales, inverses et des décrochements. Globalement, il s'agit d'une pelbiomicrosparite de texture grainstone, riche en pellets sub-arrondis et bien classé (faciès E). Ainsi, ce faciès est caractérisé par la présence des pollens indéterminés (fig. 4 : L et M), de dinoflagellés, de foraminifères benthiques (Textularia, Miliole) 
souvent micritisés, de gastéropodes, de bélemnites, de glauconies (fig. 4 : N) et de rares bioturbations.

De point de vue paléoenvironnement, le faciès du Domérien inférieur suggère la prédominance d'un environnement de type intertidal profond. La présence des pollens, des algues (Dasycladacées), plaide en faveur d'un environnement suffisamment éclairé et chaud. Généralement ces algues vivent actuellement dans des eaux où la moyenne des températures annuelles est de $20^{\circ} \mathrm{C}$ (Berger et Kaever, 1992).

\section{b- $\quad$ Formation du Domérien moyen}

Elle affleure en six séquences élémentaires; quatre transgressives prédominantes et trois régressives. La succession sédimentaire comporte, après une épaisse masse de marne grise (faciès $G$ ), d'environ 50 mètres d'épaisseur, un banc métrique de calcaire-gréseux jaunâtre. Il s'agit d'une microsparite gréseuse et azoïque (faciès $\mathrm{C}$ ), de texture grainstone, riche en quartz sub-anguleux et présentant des feldspaths et quelques rares biotite. Elle est suivie par une masse décamétrique de marne grisâtre (faciès $\mathrm{G}$ ) et de marne-sableuse blanchâtre à jaunâtre (faciès $\mathrm{H}$ ), très dure par endroit. Ensuite vient une alternance décamétrique des marnes avec des bancs métriques de calcaire gréseux de faciès $\mathrm{C}$ et avec des bancs de calcaire à calcaire-dolomitique, il s'agit d'une oobiosparite à oobiomicrite (faciès F) de texture grainstone à packstone, riche en oolithes de type sphérulites (fig.4 : D) par endroit, on note la présence de quartz et de bioturbations. Dans ce dernier faciès, la biophase est constituée principalement par des lamellibranches. A la fin de cette formation, affleure un banc décamétrique de calcaire très lité et bréchique, il s'agit d'une pelbiodolosparite (faciès E) de texture grainstone, riche en pellets et en quartz (anguleux et mal classé). La biophase est relativement abondante et très diversifiée avec des foraminifères benthiques (Miliole, Lenticulina, Textularia (fig. 4 : J)), des algues (fig.4 : C et D), des crinoïdes, des gastéropodes, des brachiopodes et des lamellibranches.

Les faciès du Domérien moyen montrent des environnements sédimentaires qui varient entre plusieurs milieux : un milieu franchement marin de type bassin profond très calme, de type infratidal très profond et supratidal profond défavorable au développement de la faune ce qui expliquerait le caractère azoïque des bancs calcaire-gréseux. Ce milieu peut être également de type intertidal peu profond très agité (bancs de calcaireoolithiques) et infratidal profond, riche en faunes, suffisamment lumineux et chaud (présence des pellets, des algues et des bryozoaires). 


\section{c- $\quad$ Formation du Toarcien inférieur}

Cette formation affleure sous forme d'une épaisse masse de marne grise (faciès $\mathrm{G}$ ), d'environ 140 mètres d'épaisseur. Ce faciès montre le retour d'un milieu franchement marin de type bassin profond très calme.
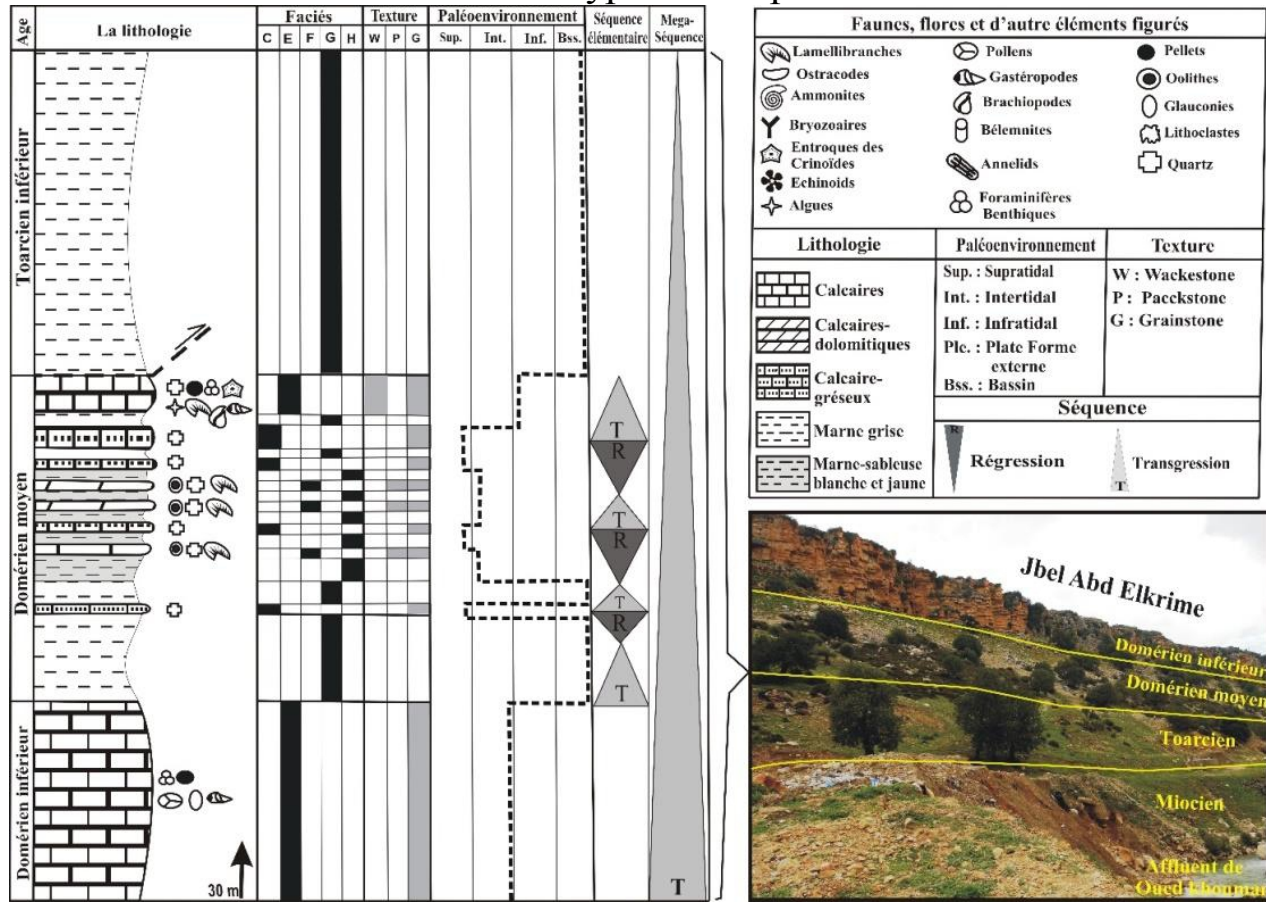

Figure 2 : Log litho-stratigraphique du Jbel Abd Elkrime

\subsection{Coupe géologique du Jbel Bin Bou Skif}

Jbel Bin Bou Skif est situé au milieu de la ride de Fert Ebir, plus vers le Nord-Ouest du Jbel Abd Elkrim. La coupe (fig.3) a été relevée dans le versant Nord-Ouest de ce Jbel, elle affleure sur plusieurs dizaines de mètres et présente trois formations sédimentaires.

\section{a- $\quad$ Formation du Domérien inférieur}

Elle se présent à la base et affleure sous forme de trois séquences élémentaires; une transgressives et deux régressives prédominantes. Sa limite supérieure est représentée par une discontinuité de ravinement bien visible. Cette formation, débute au niveau du thalweg de l'Oued Khoumane, par des bancs métriques de calcaire massif blanchâtre. La plupart de ces bancs sont surmontés et intercalés par des niveaux très dolomitiques et grisâtres. Il s'agit de biomicrite à pelbiomicrosparite (faciès B) de texture wackestone à grainstone, riche en bioturbations, en petites pellets subarrondis et en lithoclastes micritiques. La biophase est constituée 
essentiellement par des foraminifères benthiques (Textularia et Miliole), des lamellibranches, des ostracodes, des gastéropodes et des échinides.

$\mathrm{Au}$ milieu de cette formation affleurent des bancs décamétriques de calcaire lité, très bréchiques et noduleux. Au milieu et vers le haut ils alternent avec de rares niveaux de marne-sableuse jaunâtre à blanchâtre (faciès H). Il s'agit d'une pelbiomicrite à pelbiomicrosparite (faciès E) de texture packstone à grainstone, riche en pellets très mal classée et en lithoclastes micritiques. Dans ce faciès, la biophase est composée principalement par des foraminifères benthiques (Haurania deserta (fig. 4 : F), Textularia, Miliole), des lamellibranches, des crinoïdes, des bélemnites et des gastéropodes.

La partie sommitale de cette formation montre une alternance décamétrique de niveaux discontinus de marne-sableuse jaunâtre à blanchâtre (faciès $\mathrm{H}$ ) avec des bancs minces de calcaires, très bréchiques et noduleux. Il s'agit d'une oopelbiomicrite (faciès F) de texture wackestone à packstone riche en oolithes, en pellets très oxydés et mal classées par endroit, en oncolithes et en bioturbation. La biophase est constituée principalement de lamellibranches, de foraminifères benthiques (Nodosaria (fig. 4: I), Textularia, Miliole), de gastéropodes, de brachiopodes, d'algues, de crinoïdes et d'annélides (serpules).

Les faciès du Domérien inférieur montrent la prédominance de deux milieux de dépôts de type infratidal peu profond (lagon) et intertidal profond. Ils sont marqués par la présence des traces d'activité biologique (bioturbations (fig.4: K)) et l'absence d'indices d'agents mécaniques. L'abondance des bioturbations dans ces faciès suggère souvent la fréquence des arrêts et/ou des ralentissements du taux de sédimentation. En outre, l'abondance de Textularia et de Miliole indique un milieu semi-restreint à restreint et à salinité normale. La présence des algues et des annélides montre un milieu de faible turbidité avec une meilleure oxygénation. Ensuite, vers la fin de cette période, l'environnement passe à une zone intertidale peu profonde avec des dépôts d'alternance des marne-sableuses et des calcaires, qui présentent parfois des indices d'agitation plus forte (niveaux oolithiques).

\section{b- $\quad$ Formation du Domérien moyen}

Elle affleure sous forme de deux séquences élémentaires ; une régressive prédominante et une transgressive. Sa limite inférieure est représentée par une discontinuité de ravinement, par contre sa limite supérieure est représentée par un important changement de faciès marqué par le passage vers les dépôts marneux très épais. Cette formation est composée, au début et au sommet, par des bancs décamétriques et métriques de calcaire massif, ils alternent souvent avec des niveaux de marne-sableuse jaunâtre à blanchâtre (faciès $\mathrm{H}$ ). Il s'agit de biomicrite à pelbiomicrite (faciès $\mathrm{B}$ ) de 
texture wackestone, riche en bioturbations et pellets. La biophase est très rare et constituée principalement par des foraminifères benthiques (Miliole et lenticulina) et des gastéropodes.

$\mathrm{Au}$ milieu de cette formation affleurent des bancs décamétriques de calcaire-dolomitique massif. Ils sont caractérisés par une tectonique très intense avec des failles conjuguées. Il s'agit d'oopelbiomicrite à oopelbiomicrosparite (faciès F) de texture packstone à grainstone, riche en oolithes (oolithes $\alpha$, bahamithes et spherulithes), en pellets mal classés, lithoclastes micritiques et en Bioturbation. Dans ce faciès, la biophase est constituée principalement par des lamellibranches, des foraminifères benthiques (Textularia, Miliole), des gastéropodes, des brachiopodes, des algues, des ostracodes, des échinides, des crinoïdes, des annélides (serpules) et des bryozoaires.

De point de vue paléoenvironnement, les faciès du Domérien moyen suggèrent la prédominance d'un environnement de type infratidal peu profond qui passe à un milieu intertidal plus au moins profond, avec une énergie hydrodynamique très importante (des bancs calcaires riches en oolithes et litho-clastes). A la fin de cette période, le milieu passe, comme dans le Domérien inférieur, vers une zone infratidale plus profonde (des bancs calcaires riches en intercalations marneuses).

\section{c- $\quad$ Formation du Domérien supérieur}

Elle affleure sous forme de cinq séquences élémentaires ; trois transgressives prédominantes et deux régressives. Sa limite inférieure est représentée par le passage des faciès carbonatés à des faciès plus marneux. Cette formation est composée principalement par une importante masse marneuse grisâtre (faciès $G$ ), avec une centaine de mètres d'épaisseur. Elle est intercalée, au début, par des bancs métriques de calcaire-dolomie, noduleux par endroit, il s'agit d'intrabiomicrite à intrapelbiomicrite (faciès D) de texture wackestone à grainstone, riche en lithoclastes, en quartz et marque la présence de rares petits pellets. La biophase est très rare et constituée principalement par des crinoïdes et des lamellibranches. Sur ces calcaire-dolomie viennent de déposer des bancs métriques et massifs de calcaire, révélant une pelbiomicrite à pelbiomicrosparite (faciès E) de texture packstone à grainstone, riche en petites pellets sub-arrondis. Dans ce faciès, la biophase est constituée principalement de lamellibranches, d'échinides et de foraminifères benthiques (Milioles (fig. 4: $\mathrm{G}$ et $\mathrm{H}$ )). Vers le haut, des marnes alternent avec des bancs métriques de calcaires-gréseux. Il s'agit de biomicrite à céphalopodes (faciès $\mathrm{A}$ ) de texture packstone, riche en quartz, glauconie et bioturbations. La biophase est riche en macro-fossiles avec surtout des Ammonites (fig.4 : A et B), des lamellibranches, des ostracodes, des foraminifères benthiques (Textularia) et des brachiopodes. 
Les faciès du Domérien supérieur indiquent un changement radical de la sédimentation avec la prédominance d'un milieu très profond de type bassin profond très calme. Il est marqué par la prédominance des marnes grisâtres. L'intercalation de quelques bancs calcaires au milieu et vers la fin de cette période suggère le retour vers les zones proximales de type intertidal peu profond à profond. La fréquence des litho-clastes dans le premier banc de calcaire traduit une énergie hydrodynamique élevée et une augmentation du pouvoir érosif. En autre, plus vers l'Est du Jbel Bin Bou Skif, l'environnement passe vers une zone de plateforme externe (bancs de calcaire-gréseux riche en céphalopodes). C'est ainsi que la présence des calcaires lumachelliques indique la fréquence des tempêtes.

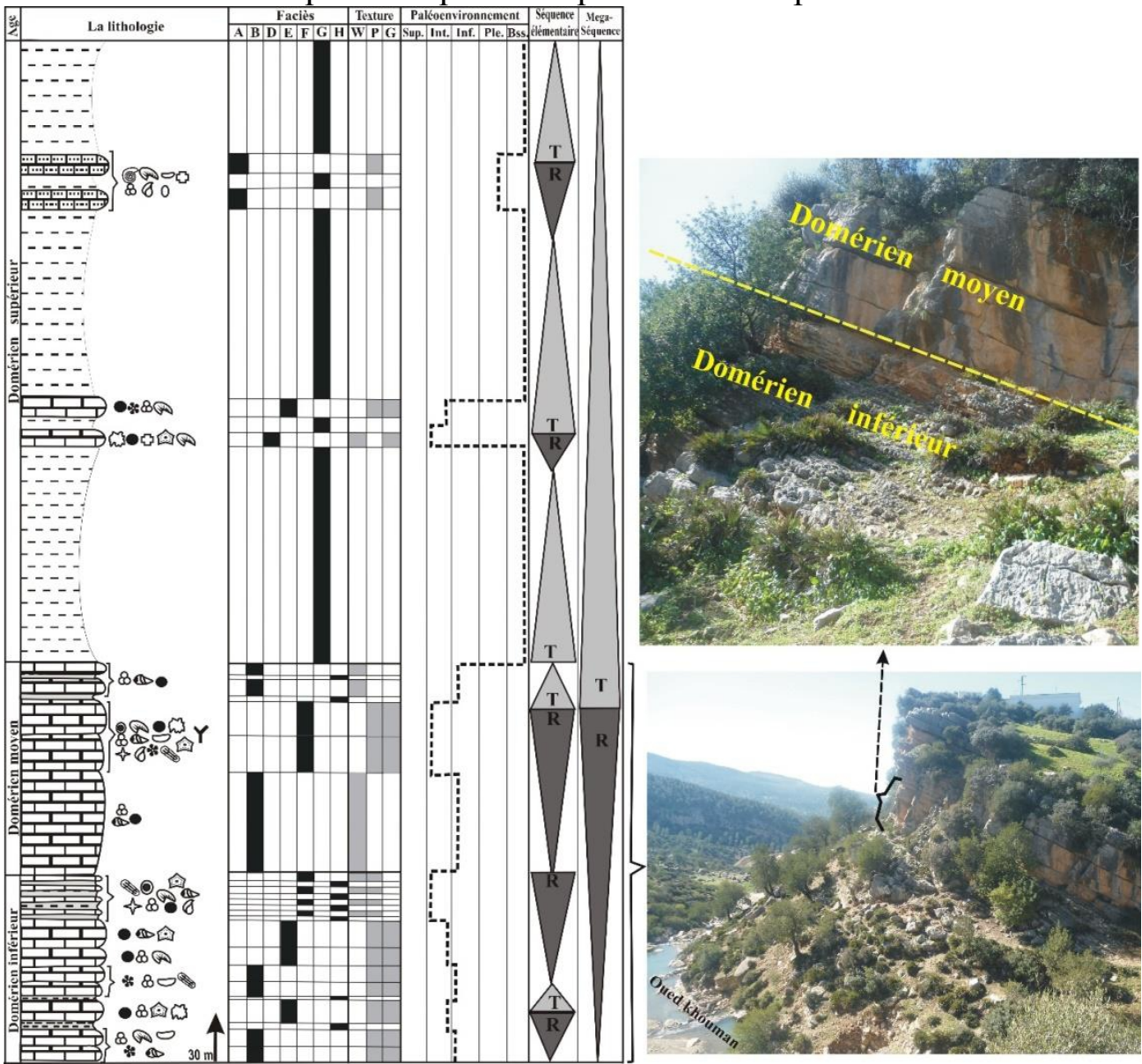

Figure 3 : Log litho-stratigraphique du Jbel Bin Bou Skif 


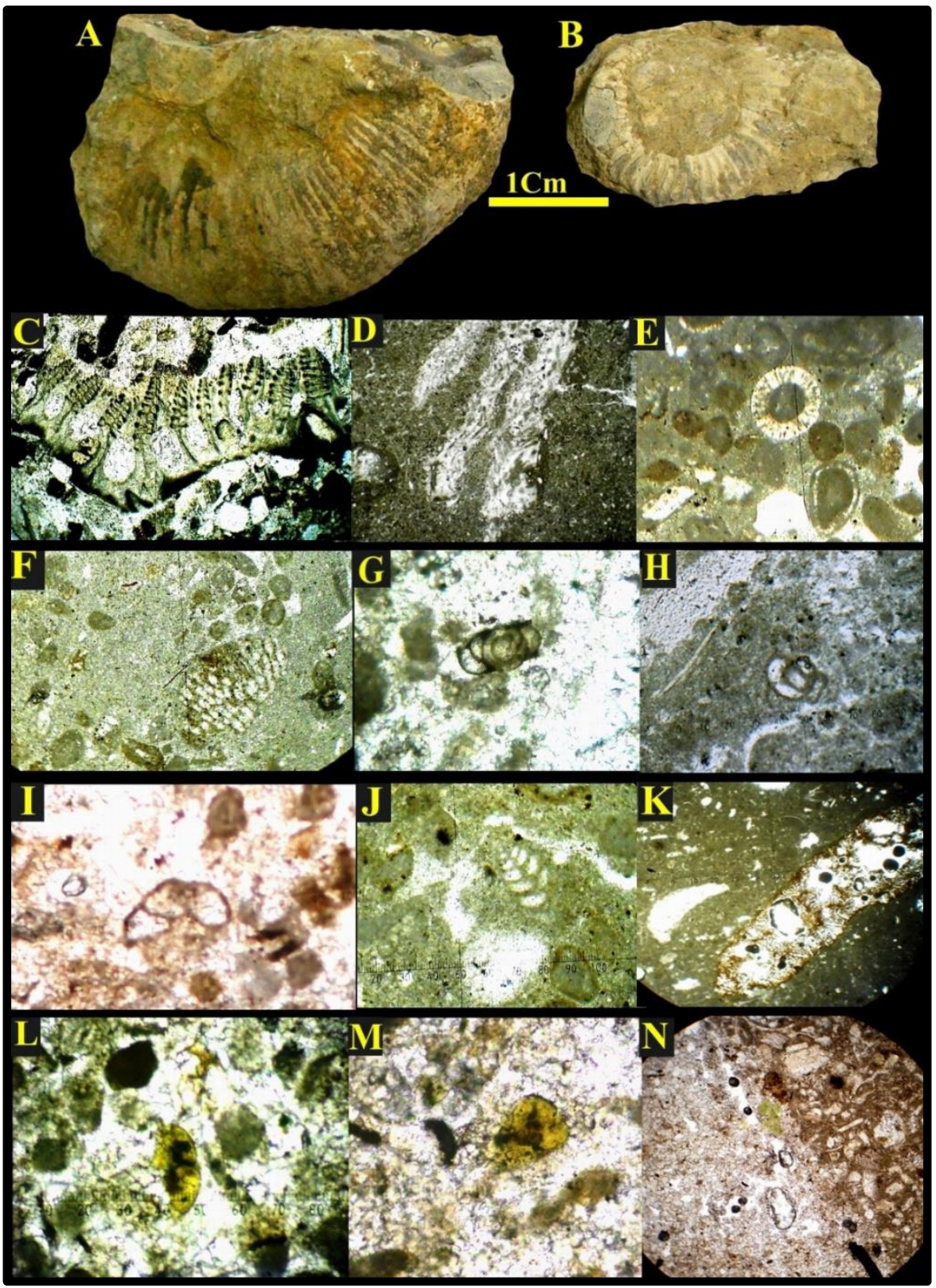

Figure 4 : Quelques photos de la faune et de la flore rencontrées dans les faciès de la ride de Fert Elbir.

A et B : Ammonites de Domérien supérieur du Jbel Bin Bou Skif. C et D : Algues du

Domérien moyen du Jbel Abd Elkrime (x40). E : Oolithes sphérulites du Domérien moyen du Jbel Abd Elkrime (x40). F : Haurania deserta de Domérien inférieur du Jbel

Bin Bou Skif (x40). G et H : Miliole du Domérien du Jbel Bin Bou Skif (x100). I :

Nodosaria du Domérien inférieur du Jbel Bin Bou Skif (x100). J : Textularia du

Domérien moyen du Jbel Abd Elkrime (x40). K : Bioturbation du Domérien inférieur du Jbel Bin Bou Skif (x40). L et M : Pollen du Domérien inférieur du Jbel Abd Elkrime (x100). N : Glauconie du Domérien inférieur du Jbel Abd Elkrime (x40). 


\section{Interprétations paléoenvironnementales et discussion}

Les analyses sédimentologiques des coupes géologiques étudiées nous permettent de proposer un schéma plus précis de l'évolution paléoenvironnementale de l'intervalle Domérien-Toarcien inférieur de la ride de Fert Elbir. La série sédimentaire identifiée révèle plusieurs types d'environnements de dépôt très diversifiés, constitués essentiellement par des milieux proximaux et distaux, situés à plusieurs centaines de mètres du rivage. Cette diversité paléoenvironnementale résulte principalement de l'interaction des facteurs externes allo-cycliques (le climat et l'eustatisme) et des facteurs internes auto-cycliques (l'hydrodynamisme, le comportement tectonique du substratum et la subsidence). Dans cette région, les paléoenvironnements sont essentiellement d'affinité téthysienne et qui montre aussi des communications très limitées avec la mer proto-Atlantique surtout au niveau du Domérien (Faugeres, 1978).

L'évolution paléogéographique montre bien la dislocation qui affecte les plates-formes carbonatées liasiques du pourtour de la Téthys occidentale durant le passage Domérien-Toarcien. Cette dislocation est un événement majeur qui est connu depuis les marges atlantiques jusqu'aux Alpes occidentales (De Graciansky et al., 1979). Elle s'est manifestée par une activité tectonique distensive responsable d'une différenciation paléogéographique très importante. Elle s'est traduite par un brusque effondrement de la plate-forme liasique et l'apparition de milieux ouverts à céphalopodes (Sadki et al. 1999). Cette activité tectonique est peut-être la responsable de la lacune sédimentaire du Domérien supérieur au niveau du Jbel Abd Elkrime, comme c'était le cas pour le Domérien supérieur de la région de Boulemane dans le Moyen Atlas marocain (Colo, 1961 ; Charrière 1990)

La corrélation (fig.5) entre les deux coupes étudiées dans cette ride montre globalement un Domérien inférieur très semblable, avec une biophase très développée et diversifiée plus vers le Nord dans la coupe du Jbel Bin Bou Skif. Par contre, le Domérien moyen de la coupe du Jbel Abd Elkrime est très épais (200m environ) et riche en marne grise et en marnesableuse par rapport à celui du Jbel Bin Bou Skif (130m environ). Ceci montre une importante subsidence du bassin dans la partie Sud durant le Domérien moyen. En outre, le Domérien supérieur affleure uniquement plus vers le Nord-Est de la coupe du Jbel Bin Bou Skif, avec des faciès très marneux présentant d'importantes épaisseurs ( $300 \mathrm{~m}$ environ), ce qui montre une importante subsidence du bassin. Les faciès principalement marneux du Toarcien-inférieur affleurent uniquement dans la coupe du Jbel Abd Elkrime, ils sont déposés directement sur le 
Log du

Jbel Bin Bou Skif

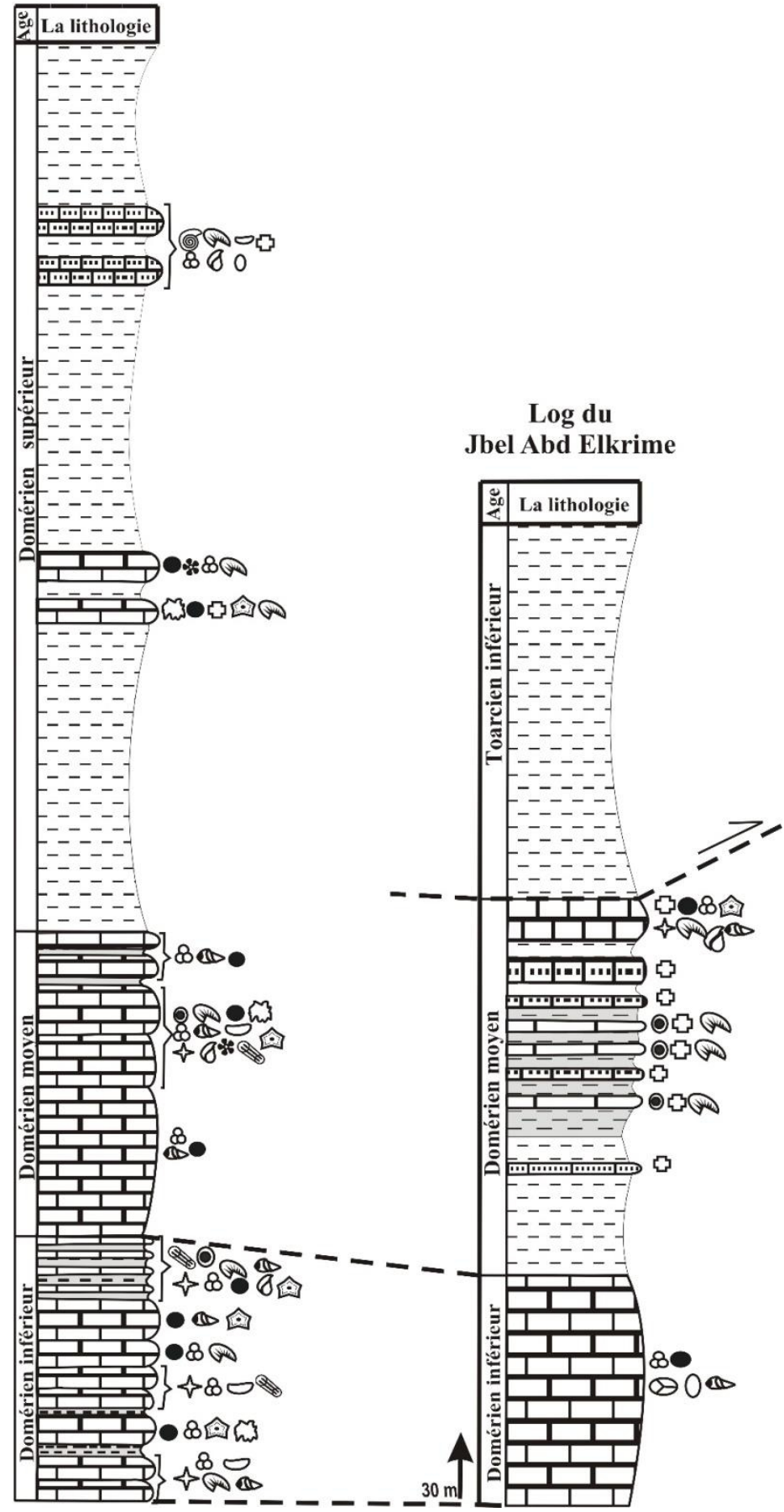

Figure 5 : Corrélation entre les deux logs lithostratigraphiques de la ride de Fert Elbir 
Domérien moyen, ce qui montre la présence d'une lacune de sédimentation ou d'érosion durant le Domérien supérieur dans la partie sud. Ces faciès (200m environ) est en faveur de cette importante subsidence du bassin.

En résumé, le domaine sud-rifain a été recouvert par une mer peu profonde durant le Domérien-inférieur et moyen. Il est caractérisé par la stabilité $\mathrm{du}$ substratum (apports détritiques terrigènes très rares), l'homogénéité des faciès (carbonates) et la généralité des milieux littoraux de faibles profondeurs (tidaux). C'est une période biostasique qui a favorisé le long développement de faciès carbonatés tidaux en énergie moyenne et sous un climat tropical sec. Cette environnement évolue au Domérien-supérieur et au Toarcien, après une remontée eustatique, en milieux plus profond de type bassin (abondance des bancs calcaires à céphalopodes et des masses marneuses,....).

D'autre part, plus vers le Nord-Est dans la ride de Dehar En Nsour, la série sédimentaire du Domérien-Toarcien inférieur est plus développée et riche en dépôts marneux, ce qui suggère un milieu de dépôt plus profond par rapport à la ride de Fert Elbir. Par conséquent, à partir de cette comparaison on peut déduire que l'espace d'accommodation du bassin de dépôt du domaine sud-rifain augmente depuis l'Ouest vers l'Est.

L'identification des foraminifères benthiques rencontrés dans la plupart des faciès du Domérien-Toarcien inférieur de la ride de Fert Elbir permet de distinguer deux formes principales : la forme des eaux chaudes caractérisées par des tests calcitiques, et la forme des eaux froides caractérisées par des tests agglutinés (Murray, 1991). Par conséquent, l'abondance de ces deux formes de foraminifère durant le Domérien inférieur, avec une légère suprématie de la forme à test agglutiné, montre la prédominance d'un environnement de faible température. Par contre, durant le Domérien moyen et supérieur, la prolifération des foraminifères à test calcitique et la diminution des foraminifères à test agglutiné, indique la prédominance d'un environnement à température chaude.

\section{Conclusion}

L'intervalle Domérien-Toarcien inférieur de la ride de Fert Elbir montre une sédimentation peut diversifiée, constituée principalement par des dépôts carbonatés et marneux. Huit associations de faciès ont été identifiées (Faciès A, B, C, D, E, F, G, H), avec la présence d'une macro et microfaune très diversifiée et abondante surtout au niveau du Domérien moyen. Ces faciès montrent des milieux paléoenvironnementaux très variés, constitués essentiellement par des zones proximales d'une plate-forme interne, au niveau du Domérien inférieur et moyen, et par des zones distales de type bassin, au niveau du Domérien supérieur et du Toarcien-inférieur. Les 
foraminifères benthiques rencontrés dans cette ride indiquent la prédominance d'un climat de faible température durant le Domérien inférieur. Par contre, le Domérien moyen et supérieur est prédominé par un climat chaud.

\section{References:}

1. Ait Brahim L. (1991). Tectoniques cassantes et états de contraintes récents du Maroc Nord. Résultats de la cinématique des plaques Afrique-Europe et du bloc d'Alboran. Thèse d'état, Univ. Mohamed V, Rabat, 233 p.

2. Berger S. et Kaever M. J. (1992). Dasycladales: an illustrated monograph of a fascinating algal order. Ed. Thieme, Stuttgart, 274 p.

3. Boutakiout, M. (1990). Les foraminifères du Jurassique des rides Sud-Rifaines et des régions voisines. Thèse de Doctorat es-Sciences. Université Claud Bernard, Lyon, $246 \mathrm{p}$.

4. Charrrière A. (1990). Héritage hercynien et évolution géodynamique alpine d'une chaine intracontinentale: le moyen Atlas au SE de Fès (Maroc). Thèse de doctorat Es. Sciences, Univ. Paul Sabatier, Toulouse III.

5. Chenakeb, M., (2004) - Carte Géologique du Maroc, Feuille Beni Ammar, échelle 1:50.000. Notes Mem., Serv., Géol., Maroc.

6. Colo G. (1961). Contribution à l'étude du Jurassique du Moyen Atlas septentrional. Notes et Mém. Serv. Maroc, $\mathrm{n}^{\circ} 139,226 \mathrm{p}$.

7. Daguin F. (1927). Contribution à l'étude de la région prérifaine (Maroc occidental). Notes et Mém. Serv. Géol., Rabat, Maroc, $\mathrm{n}^{\circ} 1$, 413p.

8. De Graciansky P.C., Bourbon M., Charpal O., De Chenet P.Y. \& Lemoine M. (1979). Genèse et évolution de deux marges continentales passives : marge ibérique de l'océan atlantique et marge européenne dans les Alpes occidentales. Bull. Soc. géol. Fr., (7), XXI, 5, p.663-674.

9. Dunham, R.J. (1962). Classification of carbonate rocks according todepositional texture. In classification of carbonates rock. A symposium American Association of Petroleum Geologists Bulletin, Tulsa, 1, p. 108-121.

10. ELF-Aquitaine (1975). Essai de caractérisation sédimentologique des dépôts carbonatés. 1) Éléments d'analyse. ELF-AQUITAINE. Centre de Recherche de Boussens et de Pau, 231 p.

11. ELF-Aquitaine (1977). Essai de caractérisation sédimentologique des dépôts carbonatés. 2) Éléments d'interprétation. ELFAQUITAINE, Centre de Recherche de Boussens et de Pau, 173 p. 
12. Embry, A., Klovan, J.E. (1971). A late devonian reef tracts on northeastern Banks Islands. N.W.T. Bulletin Canadian Petroleum Geologists, 19, p.730-781. [Terrminology for carbonates with clasts]

13. Faugeres, J.C. (1978). Les Rides Sud-rifaines. Évolution sédimentaire et structurale d'un bassin atlantico-mesogeen de la marge africaine. Thèse de doctorat d'état, Université de Bordeaux I, Bordeaux, 477p.

14. Flügel, E. (2004). Micro-facies of Carbonate Rocks: analysis, interpretation and application. Heidelberg Springer, Berlin, 976p.

15. Flügel, E. (2010). Micro-facies of Carbonate Rocks: analysis, interpretation and application. Second Edition, Heidelberg Springer, Berlin, 1006p.

16. Folk, R.L. (1959). Practical petrographic classification of limestones. American Association of Petroleum Geologists Bulletin, 43, p.1-38.

17. Haddaoui, Z. (2000). Influence de la géométrie d'un bassin Jurassique sur la propagation des chevauchements néogènes: géodynamique méso-cénozoïque des rides sud-rifaines (Maroc), Modélisation géométrique et numérique. Thèse de doctorat, Faculté des sciences, Université Mohamed V-Agdal, Rabat, 433p.

18. Lucas, G., Cros, P., Lang, J. (1976). Les roches sédimentaires. Etude microscopique des roches meubles et consolidées. Doin éditeurs, Paris, 496p.

19. Masrour, A. (2003). Les dépôts terrigènes du Jurassique moyen à supérieur dans le Rif externe et son avant-pays oriental (Maroc) : stratigraphie, sédimentologie et modélisations paléogéographiques. Thèse de doctorat es-sciences. Université Moulay Ismail. Meknès, 330 .

20. Murray J.W. (1991). Ecology and palaeoecology of benthic foraminifera. Longman Scientific \& Technical, 397 p.

21. Purser, B.H. (1980). Sédimentologie et diagénèse des carbonates néritiques récents, les éléments de la sédimentation et de la diagénèse. Publications de l'institut français du pétrole, Tome 1 et Tome 2, éditions technip, 366p.

22. Saaidi E.K. (1983). Histoire géologique du Maroc. Livre, Imprimerie Mohamed V culturelle et universitaire, Fès, $N^{\circ} 2,213 p$.

23. Sadki D., Ettaki M., Chellai E. H., Milhi A., Amhoud H. \& ElKamar A. (1999). Les événements géodynamiques sédimentaires et biologiques au passage Lias moyen-Lias supérieur dans le HautAtlas central marocain. Europ. Palaeont. Assoc. Workshop, Lisboa, Abstract, p.98-101.

24. Sani F., Del Ventisette C., Montanari D. \& al. (2007). Structural evolution of the Rides Prerifaines (Morocco): structural and seismic 
interpretation and analogue modelling experiments. International Journal of Earth Science (Geol Rundsch), 96, pp. 685-706.

25. S.C.P (1966) : Société Chérifienne des Pétroles. Le bassin du SudOuest marocain, In : Bassins sédimentaires du littoral africain, Symposium New-Delhi, 1964. publ, Ass. Serv, geol. afric., Paris, $1^{\text {ére }}$ part. (Littoral atlantique), p. 5 a 11.

26. Vernli R. (1987). Micropaléontologie du Néogène post-nappes du Maroc septentrional et description systématique des foraminifères planctoniques. Notes et Mém. Serv. Géol. Maroc, n 331, 266 p. 